\title{
Association Between Novel Coronavirus Disease 2019 (COVID-19) and Idiopathic Intracranial Hypertension
}

\author{
Ahmad Talebian ${ }^{1}$, Babak Soltani ${ }^{1,}{ }^{,}$, Dawood Aghadoost ${ }^{2}$, Javid Azadbakht ${ }^{3}$, Alireza Rezaee ${ }^{4}$ and Zahra \\ Abbasy $^{4}$ \\ ${ }^{1}$ Infectious Diseases Research Center, Kashan University of Medical Sciences, Kashan, Iran \\ ${ }^{2}$ Department of Ophthalmology, Kashan University of Medical Sciences, Kashan, Iran \\ ${ }^{3}$ Department of Radiology, Kashan University of Medical Sciences, Kashan, Iran \\ ${ }^{4}$ Medical Student Research Center, Kashan University of Medical Sciences, Kashan, Iran \\ "Corresponding author: Infectious Diseases Research Center, Kashan University of Medical Sciences, Kashan, Iran. Email: babak_soltani1969@yahoo.com \\ Received 2021 April 11; Revised 2021 May 05; Accepted 2021 May 19.
}

\begin{abstract}
Introduction: Idiopathic intracranial hypertension (IIH), also known as pseudotumor cerebri, is a rare neurological manifestation of coronavirus disease 2019 (COVID-19) infection.

Case Presentation: In this case study, we present a 10-year-old girl with headache, diplopia, bilateral sixth nerve palsy, and papilledema following a history of seven days of fever about two weeks ago. In lumbar puncture, the opening pressure was $56 \mathrm{cmH}_{2} \mathrm{O}$. Cerebrospinal fluid analysis was normal. Brain magnetic resonance imaging (MRI) was suggestive of IIH. Nasopharyngeal reverse transcription-polymerase chain reaction (RT-PCR) was positive for COVID-19. Oral acetazolamide was prescribed, and the patient improved completely after three weeks of continuous medical treatment.

Conclusions: Since IIH is a rare neurological complication of COVID-19, we have to suspect COVID-19 infection in any patient with $\mathrm{IIH}$.
\end{abstract}

Keywords: COVID-19, Pseudotumor Cerebri, Idiopathic Intracranial Hypertension, SARS-CoV-2

\section{Introduction}

Since the emergence of the novel coronavirus disease 2019 (COVID-19) pandemic, several associated neurological manifestations have been reported in the related literature. Regarding a cohort study conducted on severely ill admitted COVID-19 patients, neurological complications have been documented in $45.5 \%$ of infected patients 1 - 14 days after the onset of infection $(1,2)$. Reportedly, neurological manifestations of COVID-19, which are assumed to be caused by exaggerated immune-mediated response, are a frequent complication to be expected following infection. It seems about $36 \%$ of pediatric patients with COVID19 have neurological involvement attributable to either peripheral or central nervous systems. Sixth nerve palsy in viral infections may be due to either direct invasion or indirect immune mechanisms (3). One of the uncommon features of COVID-19 infection is IIH (4-7).

Pseudotumor cerebri is a clinical condition which presents with headache, papilledema, increased blood pressure, and normal cerebrospinal fluid analysis. There are some causes that have been attributed to these signs and symptoms, such as venous sinus thrombosis, toxicity of some medications such as tetracyclines, vitamin A, contraceptives, and sepsis $(8,9)$. Furthermore, sixth nerve palsy has been related to some viral infections in children, including enterovirus and Epstein-Barr, as well as vaccinations. Although sixth nerve palsy is not well studied in children, its incidence is low among them. Some important causes of abducens nerve palsy include multiple sclerosis (MS), vasculopathies, tumors, and inflammatory conditions such as viral infections. However, if accompanied by papilledema, it strongly suggests increased intracranial pressure (ICP) associated with IIH among COVID-19 patients (3).

Herein, we describe a 10-year-old girl with confirmed COVID-19 infection by reverse transcription polymerase chain reaction (RT-PCR) presented with headache, diplopia, severe bilateral papilledema, and sixth nerve palsy due to IIH. 


\section{Case Presentation}

A 10-year-old female referred to pediatric neurology clinic with headache and diplopia since three days ago. The patient had a history of fever, chills, myalgia, and respiratory symptoms about two weeks ago, which had recovered after a week. Her parents also had a history of COVID19 infection about two weeks ago that was confirmed by nasopharyngeal RT-PCR. The patient had no remarkable history of medical problems, including endocrinopathy, hematologic disorder, obesity (BMI $=21)$, and use of any medications.

On physical examination, the patient was completely conscious and well-developed. An ophthalmologic exam revealed severe bilateral papilledema, and in neurological examination, she had bilateral sixth nerve palsy. Motor, sensory, and cerebellar examinations were normal. Brain magnetic resonance imaging (MRI) showed no evidence of space occupying lesion or hydrocephalus, but there was evidence of bilateral optic nerve sheath hydrops, slit-like lateral ventricles, and pituitary gland compression against pituitary. Although brain MR venography showed no evidence of sinus venous thrombosis, narrowed transverse/sigmoid sinuses were indicated (Figure 1). Blood test results were included: $\mathrm{WBC}=6500 / \mathrm{mm}^{3}(\mathrm{PMN}=45 \%$, lymph $=55 \%), \mathrm{Hb}=13.5 \mathrm{~g} / \mathrm{dL}, \mathrm{PLT}=260000 / \mathrm{mm}^{3}, \mathrm{ESR}=$ $36, \mathrm{CRP}=45 \mathrm{mg} / \mathrm{L}, \mathrm{Na}=141 \mathrm{mmol} / \mathrm{L}, \mathrm{K}=4.2 \mathrm{mmol} / \mathrm{L}, \mathrm{Ca}=$ $9.5 \mathrm{mg} / \mathrm{dL}, \mathrm{P}=4 \mathrm{mg} / \mathrm{dL}, \mathrm{ALT}=35 \mathrm{IU} / \mathrm{L}, \mathrm{AST}=40 \mathrm{IU} / \mathrm{L}, \mathrm{ALP}=$ $350 \mathrm{IU} / \mathrm{L}, \mathrm{BS}=85 \mathrm{mg} / \mathrm{dL}, \mathrm{BUN}=13 \mathrm{mg} / \mathrm{dL}, \mathrm{Cr}=0.5 \mathrm{mg} / \mathrm{dL}, \mathrm{T} 4$ $=10.5 \mu \mathrm{g} / \mathrm{dl}$ (5 - 14.5), TSH = $3.5 \mathrm{mIU} / \mathrm{L}$ (0.35 - 5.1), PTH = 32 $\mathrm{Pg} / \mathrm{mL}(10$ - 65). Lumbar puncture was performed; the cerebrospinal fluid (CSF) analysis was normal, and CSF opening pressure was $56 \mathrm{cmH}_{2} \mathrm{O}$. Retinography indicated bilateral optic disc swelling, paleness, and blurred borders. Optic nerve head ocular coherence tomography (OCT) revealed papilledema and decrease in nerve fiber layer thickness in both eyes (Figure 2). Considering the history of a febrile illness in our patient and positive history of COVID-19 infection in her parents, RT-PCR for COVID-19 was obtained by nasopharyngeal swab, which was positive. CSF RT-PCR was negative for COVID-19. We ruled out other differential diagnoses of IIH by laboratory tests, ophthalmologic evaluations, and neuroimaging modalities. Due to the positive history of COVID-19 infection in parents and positive COVID-19 RT-PCR in our patient, we did not perform other virology tests.

Based on the findings on physical examination and paraclinical evaluations (OCT, retinography, brain MRI, and lumbar puncture), the most probable diagnosis was IIH. The patient was admitted for three days. Medical treat- ment with oral acetazolamide $1000 \mathrm{mg}$ daily was started. After three weeks of medical treatment, in clinical exam, we found that sixth nerve palsy was remarkably subsided, and ophthalmoscopy showed a significant remission of papilledema (Figure 3). Acetazolamide was continued about eight weeks, and the patient had no problem during this period.

\section{Discussion}

We reported a 10-year-old girl with symptoms and sings of IIH associated with a history of COVID-19 infection for about two weeks. COVID-19 infection is not just a respiratory system infection, and it can result in some extrapulmonary involvement. They can result from several mechanisms: first, in COVID-19 infection, a "cytokine storm" may develop, and pro-inflammatory cytokines are abundant in circulatory system. These cytokines can result in systemic inflammatory response and tissue injuries subsequently (10). Second, COVID-19 can result in hypercoagulable state, either by direct attachment to endothelial cells via angiotensin converting enzyme-2 (ACE-2) receptor and subsequent endothelial damage or as a result of systemic inflammatory response and activation of coagulation system. Endothelial dysfunction and hypercoagulable state change blood supply of various organs by causing vasoconstriction and thrombosis, which eventuate ischemia and tissue damage (11). Third, COVID-19 can directly attack cells causing cell injury and ultimate organ dysfunction (4).

One of the systems affected by COVID-19 is nervous system (either peripheral or central). As aforementioned, some mechanisms contribute to systemic organ dysfunction in COVID-19 infection. Neurological complication is caused by various mechanisms including neurotropic or neuroinvasive effect of severe acute respiratory syndrome coronavirus 2 (SARS-CoV-2), systemic inflammatory response, thrombotic events, and also post-infectious autoimmune effect of COVID-19 $(12,13)$. According to these mechanisms, different neurological manifestations can occur, including ageusia, anosmia, encephalopathy, vascular events, and Guillain-Barre syndrome $(14,15)$.

IIH is a condition presented with headache, bilateral papilledema, bilateral sixth nerve palsy, normal CSF profile, and a normal neuroimaging (except non-specific findings such as prominent subarachnoid space around optic nerve, vertical tortuosity of optic nerve, and superior compression of hypophysis) $(8,9)$. Other etiologies of pseudotumor cerebri include sepsis, long-term corticosteroid consumption or corticosteroid withdrawal, use of contra- 




Figure 1. A and B, Coronal fat suppressed SE T2WI. Red arrows in A indicate optic nerve sheath hydrops and yellow arrows in B show slit-like lateral ventricles; C, Midsagittal SE T2WI. Partial empty sella, as pituitary gland compressed against pituitary fossa, is noted (green arrows); D, 3D phase contrast MRV. Blue arrows delineate narrowed transverse and sigmoid sinuses bilaterally.

ceptives, tetracyclines, hypervitaminosis A, etc. $(8,16)$. The pathogenesis of IIH is not definitely known yet. The probable mechanisms include venous outflow abnormalities, raised intracranial venous pressure, altered production and absorption of CSF, low-grade inflammation, intracranial vascular clotting, and cerebral edema (17). 


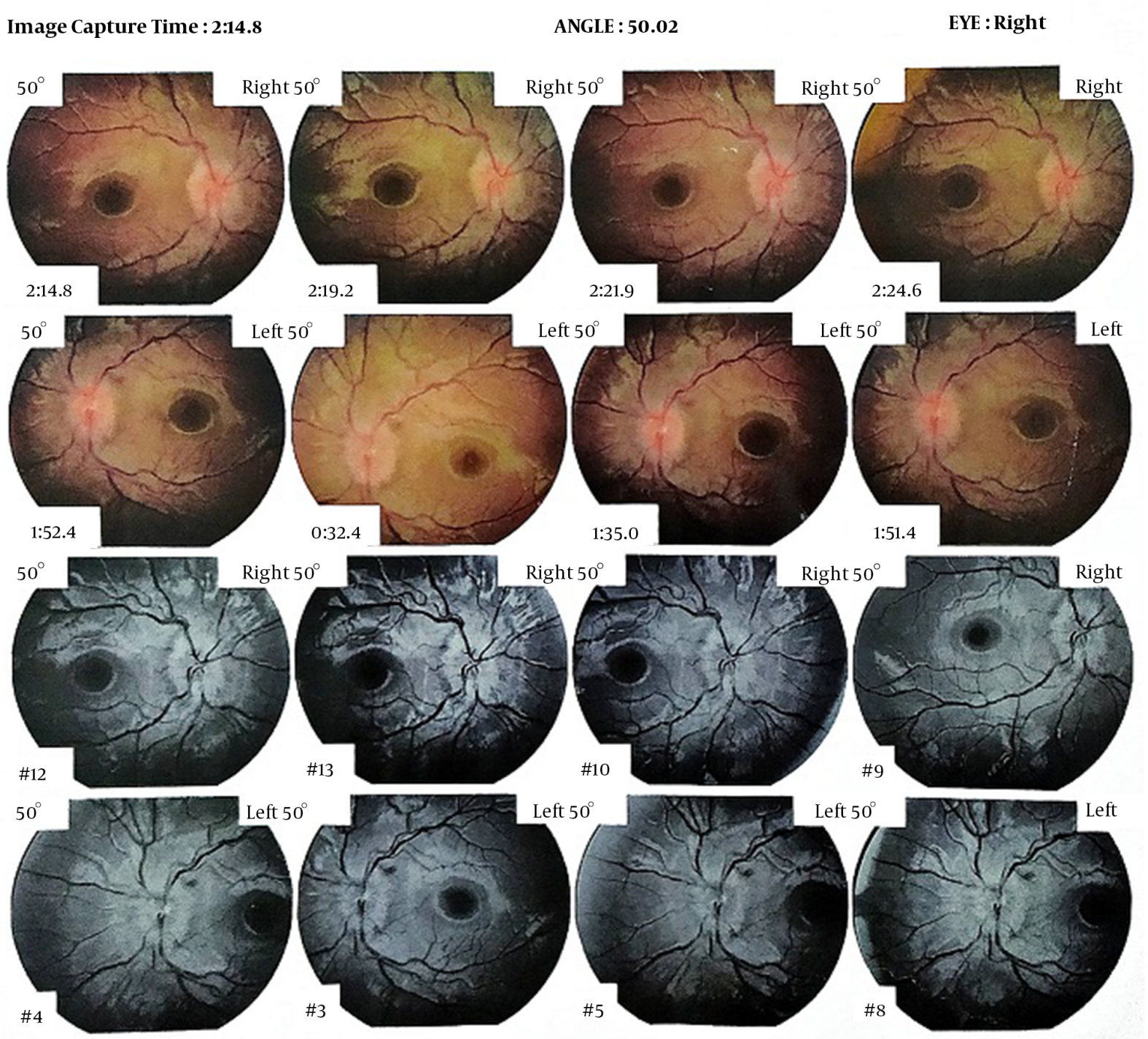

Figure 2. Retinography and fluorescein angiography of both eyes showing bilateral frank papilledema with paleness of optic disc and blurred borders accompanied by tortuosity of retinal vessels.

Mechanisms of IIH in COVID-19 infection are somehow similar to those contributing to COVID-19 neurological complications that include low-grade inflammation accompanied by hyperviscosity and hypercoagulable state (18). The patients who meet the criteria of IIH with CSF pleocytosis most likely have secondary increased ICP due to infectious agents rather than true IIH, including bacterial, fungal, tuberculous meningitis (TBM), viral encephalitis or meningitis, and Lyme disease (19). According to normal CSF profile in our case, the most probable cause of sixth nerve palsy is increased intracranial pressure due to IIH.
The main strength of our case report is complete work up of the patient, such as performing brain MRI and magnetic resonance venography (MRV), retinography and fluorescein angiography of eyes. However, the main limitation is the lack of investigating viral causes other than COVID19.

\subsection{Conclusions}

In any child with clinical manifestation of IIH we should suspect COVID-19 infection besides ruling out other etiologies. In this issue, taking the history of contact with 


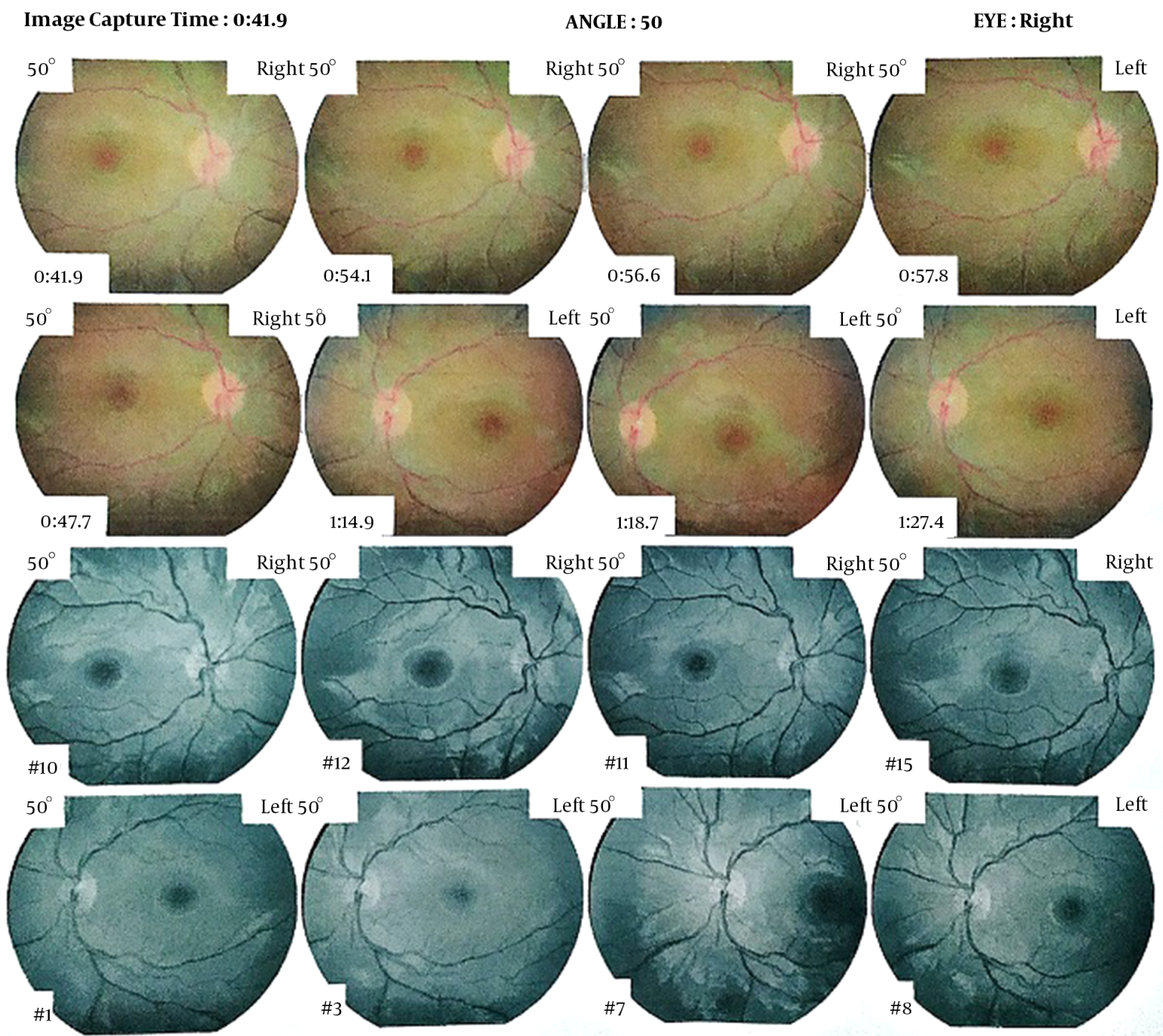

Figure 3. Retinography and fluorescein angiography of both eyes after three weeks of treatment, which indicates full remission of papilledema, defined by pinkish color and sharp border of optic disc in both eyes.

COVID-19 infected patients and performing virology tests are mandatory.

\section{Acknowledgments}

We would like to express our gratitude to the personnel of pediatric ward-2 of Shahid Beheshti Hospital, Kashan, Iran.

\section{Footnotes}

Authors' Contribution: All authors contributed equally to writing the manuscript.
Conflict of Interests: The authors declared no conflict of interests.

Funding/Support: This study received no funding.

Informed Consent: Parental consent was obtained for reporting their child.

\section{References}

1. Mao L, Jin H, Wang M, Hu Y, Chen S, He Q, et al. Neurologic Manifestations of Hospitalized Patients With Coronavirus Disease 2019 in Wuhan, China. JAMA Neurol. 2020;77(6):683-90. doi: 10.1001/jamaneurol.2020.1127. [PubMed: 32275288]. [PubMed Central: PMC7149362]. 
2. Silva MTT, Lima MA, Torezani G, Soares CN, Dantas C, Brandao CO, et al. Isolated intracranial hypertension associated with COVID-19. Cephalalgia. 2020;40(13):1452-8. doi: 10.1177/0333102420965963. [PubMed: 33146040]. [PubMed Central: PMC7645603].

3. Falcone MM, Rong AJ, Salazar H, Redick DW, Falcone S, Cavuoto KM. Acute abducens nerve palsy in a patient with the novel coronavirus disease (COVID-19). J AAPOS. 2020;24(4):216-7. doi: 10.1016/j.jaapos.2020.06.001. [PubMed: 32592761]. [PubMed Central: PMC7311910].

4. Baig AM, Khaleeq A, Ali U, Syeda H. Evidence of the COVID-19 Virus Targeting the CNS: Tissue Distribution, Host-Virus Interaction, and Proposed Neurotropic Mechanisms. ACS Chem Neurosci. 2020;11(7):9958. doi: 10.1021/acschemneuro.0c00122. [PubMed: 32167747]. [PubMed Central: PMC7094171].

5. Soto Insuga V, Cantarín-Extremera V, Solís-Muñoz I, Buendía-Martínez $\mathrm{S}$, Atencia-Ballesteros M, Bernardino B, et al. Pseudotumor Cerebri Caused by SARS-CoV-2 Infection in a Boy. J Pediatr Neurol. 2020;19(3):207-9. doi:10.1055/s-0040-1715857.

6. Stafstrom CE, Jantzie LL. COVID-19: Neurological Considerations in Neonates and Children. Children (Basel). 2020;7(9). doi: 10.3390/children7090133. [PubMed: 32927628]. [PubMed Central: PMC7552690].

7. Wu Y, Xu X, Chen Z, Duan J, Hashimoto K, Yang L, et al. Nervous system involvement after infection with COVID-19 and other coronaviruses. Brain Behav Immun. 2020;87:18-22. doi: 10.1016/j.bbi.2020.03.031. [PubMed: 32240762]. [PubMed Central: PMC7146689].

8. Corbett JJ. "Pseudotumor Cerebri" by any other name. Arch Ophthalmol.2000;118(12):1685. doi: 10.1001/archopht.118.12.1685. [PubMed: 11115265].

9. Noro F, Cardoso FM, Marchiori E. COVID-19 and benign intracranial hypertension: A case report. Rev Soc Bras Med Trop. 2020;53. e20200325. doi: 10.1590/0037-8682-0325-2020. [PubMed: 32520086]. [PubMed Central: PMC7294953].

10. Lopes-Pacheco M, Silva PL, Cruz FF, Battaglini D, Robba C, Pelosi $\mathrm{P}$, et al. Pathogenesis of Multiple Organ Injury in COVID-19 and Potential Therapeutic Strategies. Front Physiol. 2021;12:593223. doi: 10.3389/fphys.2021.593223. [PubMed: 33584343]. [PubMed Central: PMC7876335].
11. Becker RC. COVID-19 update: Covid-19-associated coagulopathy. J Thromb Thrombolysis. 2020;50(1):54-67. doi: 10.1007/s11239-020-021343. [PubMed: 32415579]. [PubMed Central: PMC7225095].

12. Atluri VS, Hidalgo M, Samikkannu T, Kurapati KR, Nair M. Synaptic Plasticity and Neurological Disorders in Neurotropic Viral Infections. Neural Plast. 2015;2015:138979. doi: 10.1155/2015/138979. [PubMed: 26649202]. [PubMed Central: PMC4663354].

13. Coperchini F, Chiovato L, Croce L, Magri F, Rotondi M. The cytokine storm in COVID-19: An overview of the involvement of the chemokine/chemokine-receptor system. Cytokine Growth Factor Rev. 2020;53:25-32. doi: 10.1016/j.cytogfr.2020.05.003. [PubMed: 32446778]. [PubMed Central: PMC7211650].

14. Alberti P, Beretta S, Piatti M, Karantzoulis A, Piatti ML, Santoro P, et al. Guillain-Barre syndrome related to COVID-19 infection. Neurol Neuroimmunol Neuroinflamm. 2020;7(4). doi: 10.1212/NXI.0000000000000741. [PubMed: 32350026]. [PubMed Central: PMC7217652].

15. Mak PQ, Chung KS, Wong JS, Shek CC, Kwan MY. Anosmia and Ageusia: Not an Uncommon Presentation of COVID-19 Infection in Children and Adolescents. Pediatr Infect Dis J. 2020;39(8):e199-200. doi: 10.1097/INF.0000000000002718. [PubMed: 32516281].

16. Kwon YJ, Allen JL, Liu GT, McCormack SE. Presumed Pseudotumor Cerebri Syndrome After Withdrawal of Inhaled Glucocorticoids. Pediatrics. 2016;137(6). doi: 10.1542/peds.2015-2091. [PubMed: 27244842]. [PubMed Central: PMC4894252].

17. Digre KB, Corbett JJ. Idiopathic Intracranial Hypertension (Pseudotumor Cerebri): A Reappraisal. Neurol. 2001;7(1):2-68. doi: 10.1097/00127893-200107010-00002.

18. Dolhnikoff M, Duarte-Neto AN, de Almeida Monteiro RA, da Silva LFF, de Oliveira EP, Saldiva PHN, et al. Pathological evidence of pulmonary thrombotic phenomena in severe COVID-19. J Thromb Haemost. 2020;18(6):1517-9. doi: 10.1111/jth.14844. [PubMed: 32294295]. [PubMed Central: PMC7262093].

19. Beal JC. Increased Intracranial Pressure in the Setting of Enterovirus and Other Viral Meningitides. Neurol Res Int. 2017;2017:2854043. doi: 10.1155/2017/2854043. [PubMed: 28491476]. [PubMed Central: PMC5405393]. 